DOI: $10.15690 /$ vramn890

В.А. Бывальев ${ }^{1,2,3,4,5}$, И.А. Степанов ${ }^{1}$, А.И. Кичигин ${ }^{1,5}$, В.В. Каныгин ${ }^{5,6}$, В.В. Ступак ${ }^{7}$

${ }^{1}$ Иркутский государственный медицинский университет, Иркутск, Российская Федерация

2 Дорожная клиническая больница на ст. Иркутск-Пассажирский, Иркутск, Российская Федерация

${ }^{3}$ Иркутский научный центр хирургии и травматологии, Иркутск, Российская Федерация

${ }^{4}$ Иркутская государственная академия последипломного образования, Иркутск, Российская Федерация

${ }^{5}$ Институт ядерной физики им. Г.И. Будкера СО РАН, Новосибирск, Российская Федерация

${ }^{6}$ Новосибирский государственный медицинский университет, Новосибирск, Российская Федерация

${ }^{7}$ Новосибирский научно-исследовательский институт травматологии и ортопедии им. Я.Л. Цивьяна, Новосибирск, Российская Федерация

\title{
Роль диффузионно-взвешенной
} магнитно-резонансной томографии в дифференциальной диагностике и

\section{прогнозировании выживаемости пациентов с метастазами в головной мозг}

Обоснование. Метастазы в головной мозг составляют до 40\% всех внутричерепных опухолей. Некоторые виды метастатических опухолей вызывают затруднения в дифференциальной диагностике, так как имеют схожие сигнальные характеристики с другими патологическими образованиями при нейровизуализации. Очевидным является использование дополнительных методов диагностики для определения прогноза и тактики дальнейшего ведения данной группы пациентов. Цель исследования - изучение роли диффузионновзвешенной магнитно-резонансной томографии (МРТ) в дифференциальной диагностике и прогнозировании выживаемости пациентов с метастатическими опухолями головного мозга. Методы. В исследование включены данные МРТ и патоморфологических исследований 23 пациентов с метастазами в головной мозг. Полученные значения измеряемого коэффициента диффузии (ИКД) опухолей сопоставляли с их гистологическим типом, клеточной плотностью и индексом пролиферативной активности Кі-67. Кроме того, проводили оценку влияния значений ИКД на общую выживаемость пациентов. Результаты. Установлена достоверная обратная корреляционная зависимость значений ИКД и индекса пролиферативной активности Кi-67 для различных типов метастатических опухолей головного мозга $(r=-0,774, p=0,014)$. Показана зависимость значений ИКД и общей выживаемости пациентов с метастазами в головной мозг. Общая выживаемость пациентов со значением ИКД опухоли более 947,2 мм²/сек составила 9,8 мес (95\% доверительный интервал 8,6-11,3), а при ИКД метастазов в головной мозг менее 947,2 мм²/сек-6,4 мес (95\% ДИ 3,7-9,1). Заключение. Методика диффузионно-взвешенной МРТ играет важную роль в дифференциальной диагностике метастазов в головной мозг, может использоваться в комплексной оценке предоперационного планирования хирургического лечения, а также в качестве прогностического фактора общей выживаемости для данной группы пациентов.

Ключевые слова: метастазы в головной мозг, диффузионно-взвешенная МРТ, измеряемый коэффициент диффузии, клеточная плотность, индекс пролиферативной активности Кі-67, общая выживаемость.

(Для цитирования: Бывальцев В.А., Степанов И.А., Кичигин А.И., Каныгин В.В., Ступак В.В. Роль диффузионно-взвешенной магнитно-резонансной томографии в дифференциальной диагностике и прогнозировании выживаемости пациентов с метастазами в головной мозг. Вестник РАМН. 2017;72 (6):442-449. doi: 10.15690/vramn890)

\section{Введение}

Согласно данным мировой литературы, заболеваемость метастазами в головной мозг составляет 8,3 случая на 100000 населения в год [1]. Метастатическое поражение головного мозга встречается в $37 \%$ случаев. При этом метастазы в головной мозг встречаются в $40 \%$ случаев всех внутричерепных опухолей [2]. Диагностика данной группы опухолей, как правило, не вызывает затруднений и основана на анализе клинико-неврологического обследования пациента и данных магнитно-резонансной томографии (МРТ) с проведением контрастного усиления. Тем не менее некоторые виды метастатических опухолей вызывают затруднения в дифференциальной диагностике, так как имеют схожие сигнальные характеристики на стандартных Т1- и Т2-взвешенных изображениях (T1-, Т2-ВИ) с глиомами высокой степени злокачественности, лимфомами, абсцессами, псевдотуморозной формой рас- сеянного склероза и подострым инфарктом вещества головного мозга $[3,4]$.

Доказано, что гистологический тип опухоли головного мозга влияет на интенсивность сигнала, получаемого на Т1- и Т2-ВИ $[5,6]$. Более того, внутриопухолевые изменения (кровоизлияние, некроз, формирование кистозных полостей) также способны приводить к изменению интенсивности сигнала. Известно несколько исследований, посвященных изучению роли диффузионной-взвешенной МРТ (ДВ МРТ) в дифференциальной диагностике различных опухолей головного мозга [7-17]. ДВ МРТ - это инструментальный метод, позволяющий получать изображения опухолевой ткани, взвешенные по диффузии свободных молекул воды на клеточном уровне. При этом степень диффузии молекул воды можно оценить с помощью численного значения в виде измеряемого коэффициента диффузии (ИКД). Множество значений ИКД для данной биологической структуры представляет 
собой функциональную карту диффузионно-взвешенных изображений (ДВИ) [18].

Исследования, посвященные применению ДВ МРТ при опухолях головного мозга, в том числе при метастазах, немногочисленны. Ряд авторов отмечает, что для определенных гистологических типов опухолей характерна корреляционная зависимость между значением ИКД, их клеточной плотностью и значением индекса пролиферативной активности Кі-67 [9, 10, 19]. Другие авторы в своих работах данной зависимости не отмечают [11, 12]. Учитывая высокую частоту встречаемости метастазов в головной мозг и их морфологическое разнообразие, представляется необходимым верифицировать данное заболевание уже на этапе выполнения первичного нейровизуализационного исследования с целью дальнейшего планирования вида, объема хирургического и/или радиохирургического вмешательства, а также выбора адекватной лечебной тактики ведения данной группы пациентов.

Известно, что общая выживаемость пациентов является одним из важнейших критериев в современной нейроонкологии. Под термином «общая выживаемость» принято понимать время от момента начала исследования (рандомизации, включения в исследование, начала приема препарата и т.п.) до смерти пациента от любой причины. Стоит отметить, что общая выживаемость пациентов представляет собой основную конечную точку при проведении крупных рандомизированных клинических исследований. Анализ данных мировой литературы показал наличие лишь нескольких работ, посвященных изучению зависимости между значениями ИКД и общей выживаемостью пациентов со злокачественными опухолями головного мозга (глиомами высокой степени злокачественности и метастатическими опухолями), тем не менее результаты данных исследований во многом противоречивы [13-17, 19-22].

Цель исследования - оценить роль ДВ МРТ в дифференциальной диагностике и прогнозировании выживаемости пациентов с метастатическими опухолями головного мозга путем сопоставления полученных значений ИКД опухолей с их гистологическим типом, клеточной плотностью и индексом пролиферативной активности Кі-67.

\section{Методы}

\section{Дизайн исследования}

Выполнено моноцентровое ретроспективное когортное исследование.

\section{Критерии соответствия}

В исследование включены случаи одиночных метастазов в головной мозг, подтвержденные данными патоморфологического исследования.

Критериями исключения из исследования являлись случаи множественных метастазов в головной мозг, а также пациенты после проведенных курсов химио- и радиотерапии.

\section{Условия проведения}

Исследование выполнено на базе Центра нейрохирургии НУЗ «Дорожная клиническая больница на станции Иркутск-Пассажирский» ОАО «РЖД-Медицина» (Иркутск, Российская Федерация).

\section{V.A. Byvaltsev ${ }^{1,2,3,4,5}$, I.A. Stepanov ${ }^{1}$, A.I. Kichigin ${ }^{1,5}$, V.V. Kanigin ${ }^{5,6}$, V.V. Stupak ${ }^{7}$ \\ ${ }^{1}$ Irkutsk State Medical University, Irkutsk, Russian Federation \\ ${ }^{2}$ Railway Clinical Hospital on the station Irkutsk-Passazhirskiy of Russian Railways Ltd., Irkutsk, Russian Federation \\ ${ }^{3}$ Irkutsk Scientific Center of Surgery and Traumatology, Irkutsk, Russian Federation \\ ${ }^{4}$ Irkutsk State Academy of Postgraduate Education, Irkutsk, Russian Federation \\ ${ }^{5}$ Institute of Nuclear Physics n.a. G.I. Budker of the SB RAS, Novosibirsk, Russian Federation \\ ${ }^{6}$ Novosibirsk State Medical University, Novosibirsk, Russian Federation \\ ${ }^{7}$ Novosibirsk Research Institute of Traumatology and Orthopaedics n.a. Ya. L. Tsivyan, Novosibirsk, Russian Federation}

\section{The Role of Diffusion-weighted MRI in Differential Diagnosis and Prediction of Survival in Patients with Brain Metastases}

Background: Brain metastases are observed in up to $40 \%$ of all intracranial tumors. Some types of metastatic tumors cause difficulties in differential diagnosis, since they have similar signal characteristics with other pathological entities in neuroimaging. Obviously, the additional diagnostic methods to determine the prognosis and tactics of further management of this group of patients should be implemented. Aim: To study the role of diffusion-weighted magnetic resonance imaging (MRI) in differential diagnostics and predicting the survival rate in patients with brain metastases. Materials and methods: The study included data from MRI and morphological studies of 23 patients with brain metastases. The obtained values of the apparent diffusion coefficient $(A D C)$ of tumors were compared with their histological type, cell density, and the index of proliferative activity $K i-67$. In addition, the influence of ADC values on the overall survival rate was assessed. Results: $A$ reliable inverse correlation of $A D C$ values and the index of proliferative activity for various types of brain metastases $(r=-0.74, p=0.014)$ was established. The dependence of $A D C$ values and overall survival rate of patients with metastases in the brain is presented. The overall survival rate in patients with an ADC value greater than $947.2 \mathrm{~mm}^{2} / \mathrm{sec}$ was 9.8 months (95\% CI: 8.6-11.3), and with ADC value less than $947.2 \mathrm{~mm}^{2} / \mathrm{sec}-6.4$ months (95\% CI: 3.7-9.1). Conclusion: The technique of diffusion-weighted MRI plays an important role in the differential diagnosis of brain metastases; it can be used as a tool of comprehensive preoperative assessment when planning the surgery and as a prognostic factor of overall survival rate for this group of patients.

Key words: brain metastases, diffusion-weighted MRI, apparent diffusion coefficient, cell density, Ki-67 proliferative activity index, overall survival. (For citation: Byvaltsev VA, Stepanov IA, Kichigin AI, Kanigin VV, Stupak VV. The Role of Diffusion-weighted MRI in Differential Diagnosis and Prediction of Survival in Patients with Brain Metastases. Annals of the Russian Academy of Medical Sciences. 2017;72 (6):442449. doi: 10.15690/vramn890) 


\section{Продолжительность исследования}

Исследование проводилось в период с июля 2015 по август 2017 г.

\section{Описание медицинского вмешательства}

Всем пациентам в предоперационном периоде выполнялось МРТ-исследование головного мозга с внутривенным контрастированием в различных режимах. Во всех случаях удаление опухолей выполнено одной хирургической бригадой с максимально возможной степенью радикальности при помощи микронейрохирургической техники и под увеличением операционного микроскопа OPMI Pentero 900 (Carl Zeiss, Германия). При удалении метастазов в головной мозг во всех случаях использованы флуоресцентная навигация с препаратом 5-аминолевулиновой кислоты Аласенс (НИОПИК, Россия) в режиме визуализации Blue-400, согласно современным клиническим рекомендациям [23], а также система интраоперационной нейронавигации CURVE (Brainlab, Германия). Удаленные объемные образования подвергались патоморфологическому исследованию. После получения данных МРТ-исследования и патоморфологического заключения, последние сопоставлялись между собой. Общая выживаемость пациентов оценивалась от момента включения пациента в исследование и до смерти от любой причины.

\section{Исходы исследования}

Основной исход исследования

Определены значения ИКД, индексов Кi-67 и клеточной плотности для различных гистологических типов метастатических опухолей головного мозга, а также корреляционная зависимость между указанными параметрами.

\section{Дополнительные исходы исследования}

В результате проведенного исследования выявлено, что ИКД является достоверным прогностическим фактором выживаемости пациентов с метастазами в головной мозг.

\section{Методы регистрации исходов}

МРТ-граммы (Т1-, Т2-ВИ и ДВИ) для всех пациентов, включенных в исследование, получены с помощью аппарата MPT Siemens Magnetom Essenza 1,5 T (Германия) до и после введения контрастного вещества Ультравист (Bayer Schering Pharma AG, Германия) (рис. 1, A-B). При исследовании в Т1-ВИ использовались следующие параметры: матрица $384 \times 387$, TR (время повторения) -650 , TE (время эхо) - 9,6, NEX (число возбуждений) - 1, толщина среза -4 мм, FOV (поле зрения) $-30 \times 30$. Для Т2-ВИ: матрица $384 \times 288$, TR - 4000, TE - 43, NEX - 1 , толщина среза -4 мм, FOV $-30 \times 30$. Для получения ДВИ использовался следующий набор параметров опции ДВ MPT с SE-эхо-планарным изображением (EPI): матрица $160 \times 128$, TR -7500 , TE -83 , NEX - 6, толщина среза - 4 мм, FOV - 30×30. Использованы значения $b$-фактора равные 400 и 800 сек/мм². Время сканирования в среднем составило 6 мин 30 сек.

ИКД вычислялся на нескольких ДВИ с наибольшими диаметрами объемного образования, в область интереса не были включены кистозные и некротические зоны опухолей. Подсчет ИКД производился с помощью программы RadiAnt DICOM Viewer.

Все удаленные объемные образования исследованы опытными патоморфологами. При морфологическом исследовании метастатических опухолей головного мозга оценивались следующие параметры: гистологический тип опухоли, значение индекса пролиферативной активности Кі-67 с помощью моноклональных антител МІВ-1
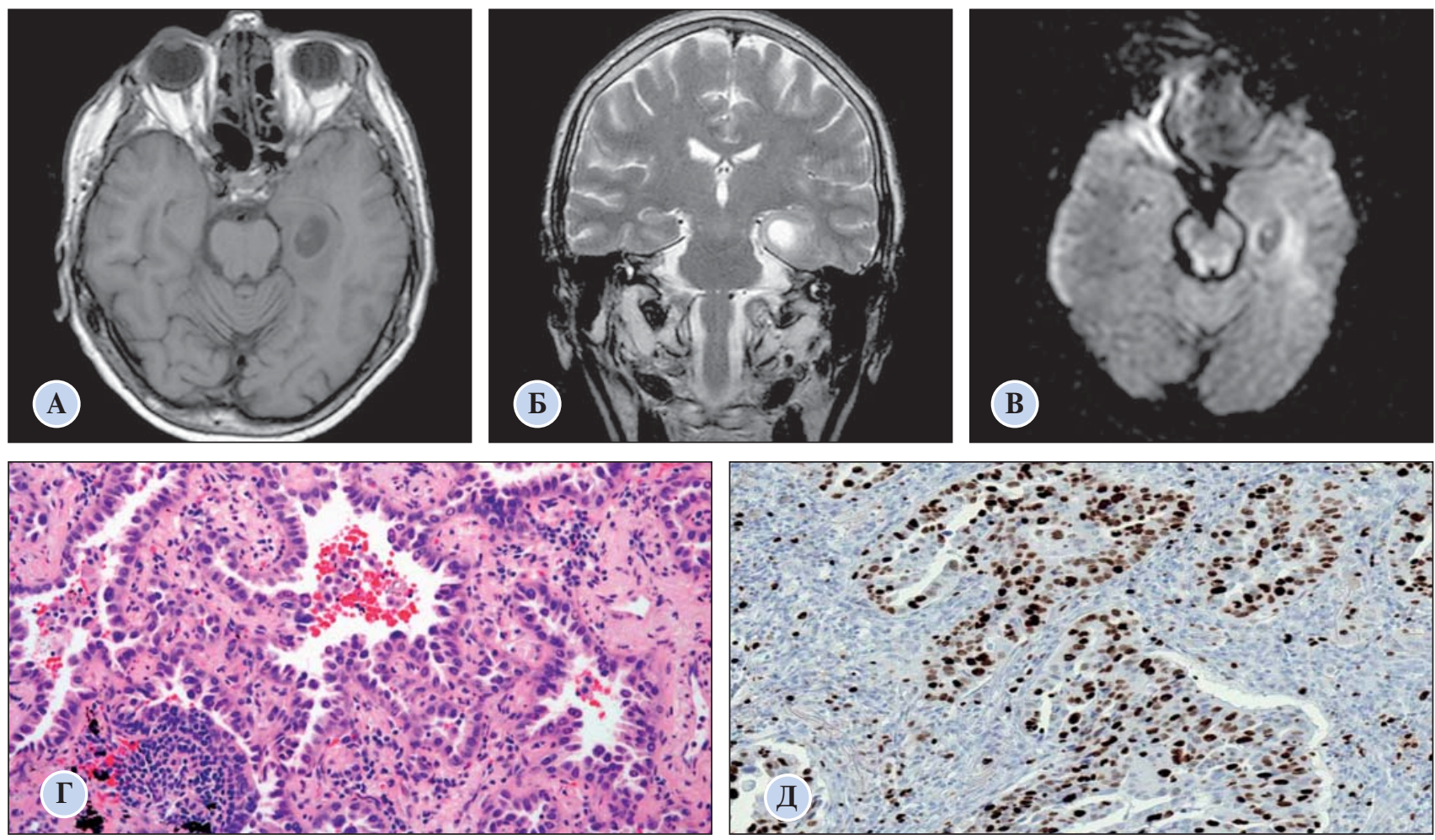

Рис. 1. МРТ и патоморфологическая картина метастаза аденокарциномы легкого в медиобазальную область левой височной доли

Примечание. А - Т1-ВИ, аксиальный срез; Б - Т2-ВИ, фронтальный срез; В - диффузионно-взвешенные изображения, измеряемый коэффициент диффузии равен 864 мм²/сек; Г - световая микроскопия, окраска гематоксилином-эозином, картина метастаза папиллярной аденокарциномы, клеточная плотность - 1239 кл./мм ${ }^{3}$; Д - окраска моноклональными антителами МІВ-1, индекс пролиферативной активности Кi-67 - 37\%. 
Таблица. Гистологические типы метастазов в головной мозг

\begin{tabular}{|c|c|c|}
\hline Гистологический тип & Число случаев, $n$ & Источник метастазирования \\
\hline Мелкоклеточный рак & 3 & Легкое \\
\hline Папиллярная аденокарцинома & 2 & Легкое \\
\hline Перстневидноклеточный рак & 1 & Легкое \\
\hline Немелкоклеточный рак & 6 & Легкое \\
\hline Тубулярная аденокарцинома & 1 & Молочная железа \\
\hline Плоскоклеточный рак & 2 & Молочная железа \\
\hline Веретеноклеточная меланома & 1 & Кожа \\
\hline Эпителиоидная меланома & 1 & Кожа \\
\hline Почечно-клеточный рак & 2 & Почка \\
\hline Светлоклеточная аденокарцинома & 1 & Почка \\
\hline Стромальная саркома & 1 & Яичник \\
\hline
\end{tabular}

(DakoCytomation, Дания), а также клеточная плотность опухолевой ткани (рис. 1, Г, Д). Подсчет клеточной плотности осуществляли с применением программы Image J при общем увеличении микроскопа AxioLab (Carl Zeiss, Германия) в 400 раз по методу М. Abercrombie [24], зна-

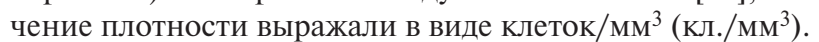

\section{Этическая экспертиза}

Протокол исследования одобрен этическим комитетом ФГБОУ ВО «Иркутский государственный медицинский университет» (протокол № 7 от 10.02.17). Исследование проводилось в соответствии с принципами надлежащей клинической практики и Хельсинкской декларации. Перед началом исследования пациенты предоставили письменное информированное согласие.

\section{Статистический анализ}

Статистическую обработку данных проводили с помощью программного обеспечения Microsoft Excel 2010. Bce измерения проверены на нормальность с помощью теста Колмогорова-Смирнова. Полученные данные оценены с помощью методов описательной статистики (абсолютных и относительных величин). Категориальные переменные выражены в процентах. Анализ общей выживаемости проводили с использованием нескорректированной модели регрессионного анализа пропорциональных рисков Кокса [25] для оценки 95\% доверительных интервалов (95\% ДИ). Кривые Каплана-Мейера применяли для оценки общей выживаемости [26]. Сравнительный анализ значений ИКД, индексов Кi-67 и клеточной плотности выполнен с помощью U-теста Манна-Уитни. Для оценки корреляционной зависимости между значением ИКД опухолей, клеточной плотностью и значением индекса Кi-67 использован коэффициент Спирмена. Порог значимости $p$ выбран равным 0,05 .

\section{Результаты}

\section{Участники исследования}

В исследование включены МРТ-граммы с ДВИ 23 пациентов с метастатическими опухолями головного мозга, прооперированных в Центре нейрохирургии ЧУЗ «Дорожная клиническая больница на ст. Иркутск-Пассажирский» ОАО «РЖД-Медицина» в период с июля 2015 по август 2017 г. Среди пациентов было 12 женщин и 11 мужчин, возраст которых варьировал от 29 до 74 лет (средний возраст составил $54,7 \pm 11,3$ года). Гистологические типы опухолей представлены в табл.

\section{Основные результаты исследования}

При сравнении средних значений ИКД различных типов метастатических опухолей отмечено достоверное различие $(p<0,01)$ (рис. 2). При этом наибольшее

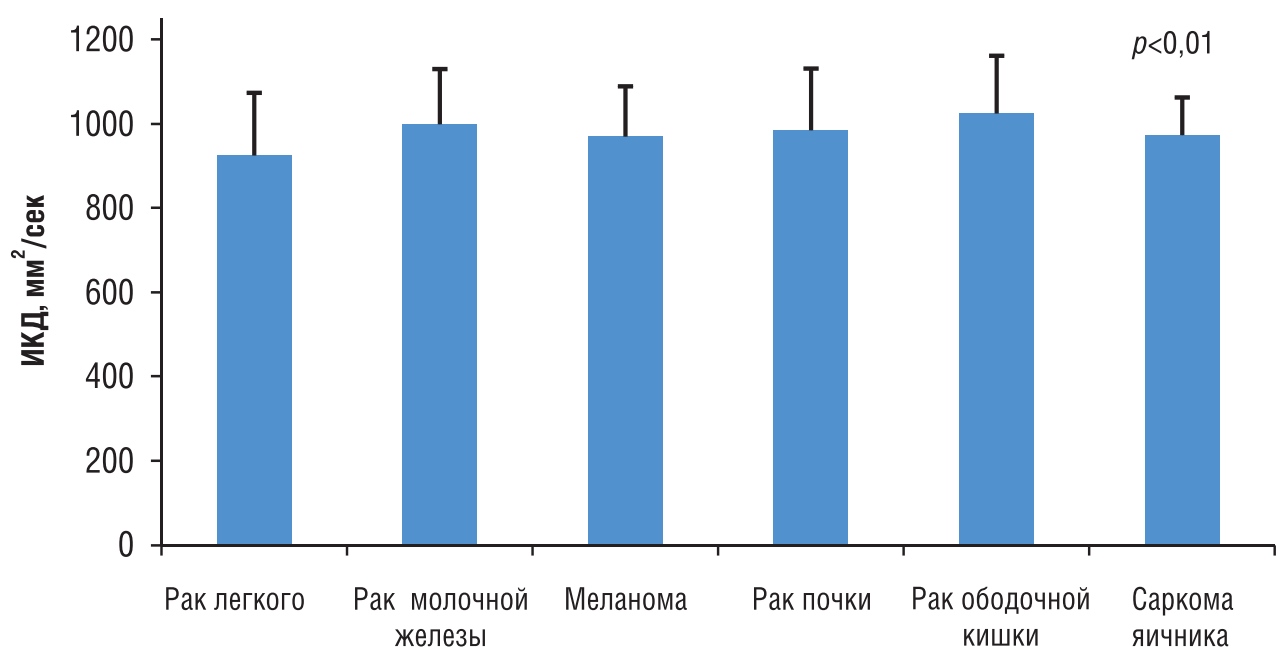

Рис. 2. Средние значения ИКД различных типов метастатических опухолей головного мозга 


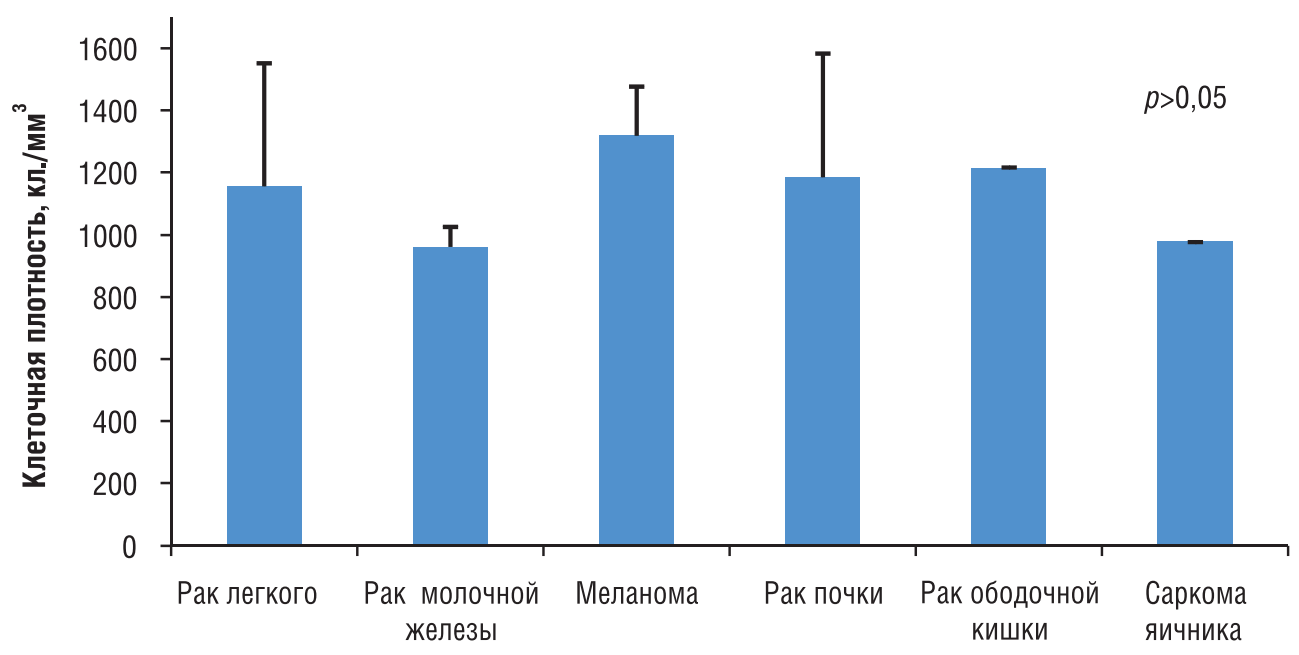

Рис. 3. Средние значения клеточной плотности в различных типах метастатических опухолей головного мозга

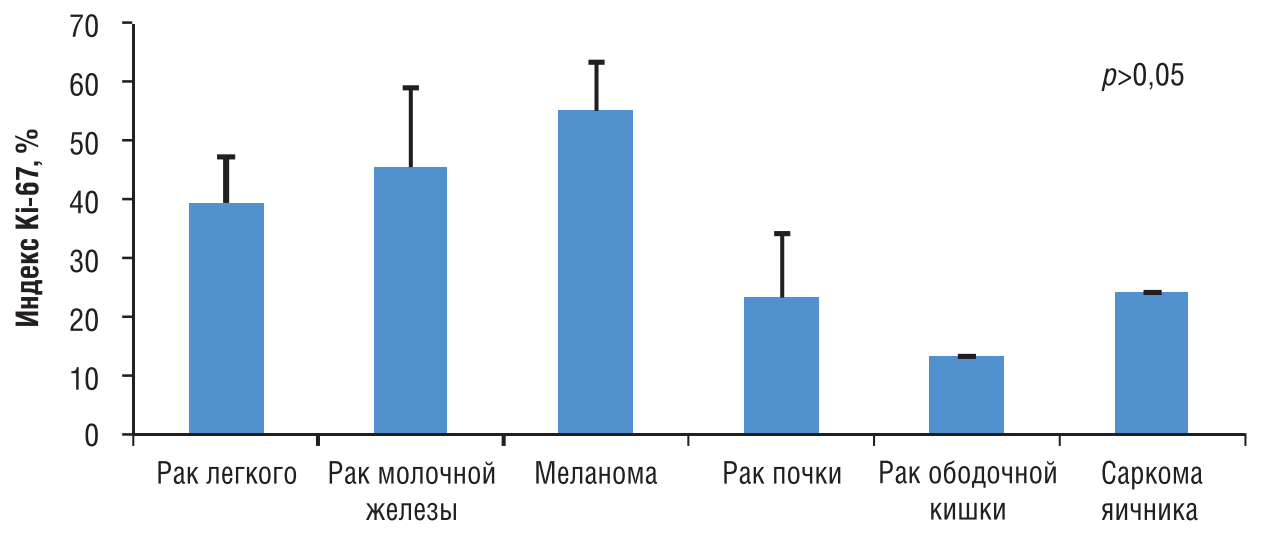

Рис. 4. Средние значения индекса пролиферативной активности Кі-67 различных типов метастатических опухолей головного мозга

среднее значение ИКД имели метастазы аденокарциномы молочной железы, почки и восходящей ободочной

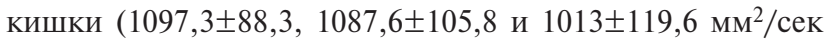
соответственно). Наименьшие средние значения ИКД отмечены в случаях метастазов в головной мозг меланомы кожи и немелкоклеточного рака легкого $(714 \pm 103,3$

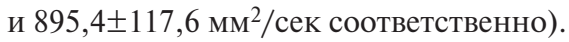

Средние значения клеточной плотности метастатических опухолей головного мозга различных гистологических типов составили для метастазов злокачественных опухолей легкого $1156,2 \pm 396,1$ кл./мм³ ${ }^{3}$ молочной желе-

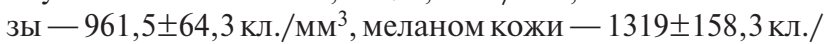

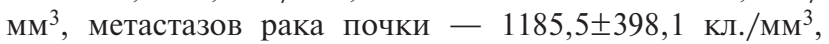
аденокарциномы ободочной кишки - 1217 кл./мм ${ }^{3}$, саркомы яичника - 977 кл./мм ${ }^{3}$. При сравнении средних значений клеточной плотности различных типов метастатических опухолей головного мозга статистически значимых различий не отмечено $(p>0,05)$ (рис. 3).

Средние значения индекса пролиферативной активности Ki-67 для различных типов метастатических опухолей варьировали в широких пределах: для метастазов

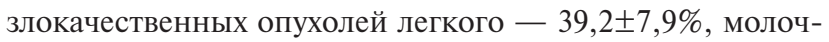

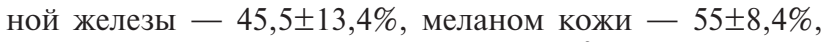

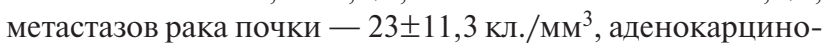
мы ободочной кишки - 27\%, саркомы яичника - 39\%. Сравнение средних значений индекса пролиферативной активности различных типов метастазов в головной мозг также не показало достоверных различий $(p>0,05)$ (рис. 4).

Анализ корреляционной зависимости между значениями ИКД и клеточной плотностью различных типов метастатических опухолей головного мозга показал отсутствие статистически значимой зависимости $(r=0,322 ; p=0,251)$.
При оценке корреляции между ИКД и значениями индекса пролиферативной активности Кі-67 отмечена достоверная обратная зависимость $(r=-0,774 ; p=0,014)$ (рис. 5).

\section{Дополнительные результаты исследования}

Оценка влияния значений ИКД на общую выживаемость пациентов показала: при значении ИКД метастатических опухолей головного мозга более 947,2 мм²/ $^{2}$ сек общая выживаемость пациентов составила в среднем 9,8 мес (95\% ДИ 8,6-11,3), при значении ИКД менее 947,2 мм²$^{2}$ сек общая выживаемость данной группы пациентов достоверно ниже и в среднем составила 6,4 мес (95\% ДИ 3,7-9,1; $p=0,019)$. Общая выживаемость пациентов в зависимости от значения ИКД метастатических опухолей представлена на рис. 6.

\section{Обсуждение}

Доказано, что значения ИКД коррелируют с клеточной плотностью и гистологическим типом различных интра- и экстракраниальных опухолей, тем самым косвенно отражая гисто- и цитоархитектонику последних [20, 21, 27-29]. К примеру, для анапластических астроцитом и глиобластом низкие значения ИКД соответствуют зонам с высокой клеточной плотностью и преобладанием цитоплазмы над ядерным компонентом клетки [20, 29]. Для некоторых злокачественных экстракраниальных опухолей (рак легкого, молочной железы и прямой кишки) также имеет место корреляционная зависимость между низкими значениями ИКД и степенью дифференциации $[20,21]$. В отношении метастатических опухолей голов- 


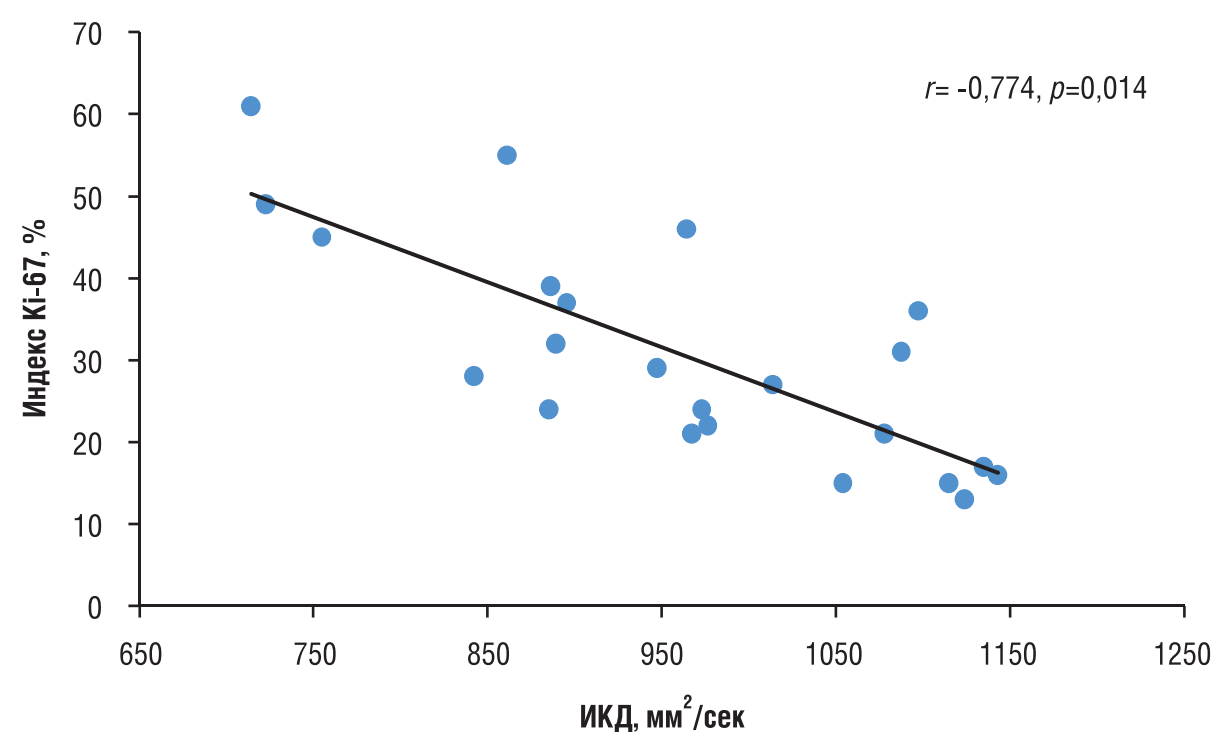

Рис. 5. Анализ корреляционной зависимости между значениями ИКД и индексом пролиферативной активности Кі-67 метастатических опухолей головного мозга: кривая корреляции демонстрирует достоверную обратную зависимость

Примечание. ИКД — измеряемый коэффициент диффузии.

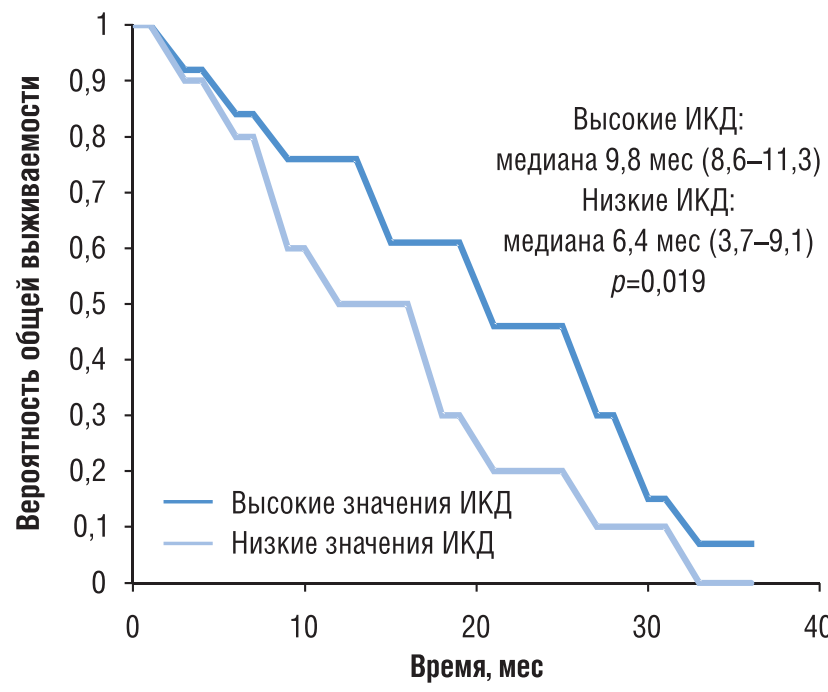

Рис. 6. Кривые Каплана-Мейера для низких и высоких значений ИКД показывают общую выживаемость пациентов с метастатическими опухолями головного мозга

Примечание. ИКД — измеряемый коэффициент диффузии.

ного мозга показано, что низкие значения ИКД ассоциированы с высокой клеточной плотностью и низкой дифференцировкой опухолей [7, 8]. Нами не обнаружено достоверной корреляционной зависимости между значениями ИКД и клеточной плотностью метастазов в головной мозг. Мы считаем, что полученные низкие значения ИКД для некоторых опухолей связаны с особенностями их биологии и микроструктурного строения. Богатая коллагеновыми и ретикулиновыми волокнами опухолевая строма значительно ограничивает диффузию свободных молекул воды, что в конечном итоге находит свое отражение в низких значениях ИКД. Этого же мнения придерживаются и некоторые исследователи [30, 31].

Как известно, ядерный белок Кі-67 определяет степень пролиферативной активности клеток, что является важнейшим моментом в определении агрессивности опухоли. Максимальный уровень белка Кі-67 в клетке регистрируется в митозе. В G1-фазе клеточного цикла про- исходит падение его уровня, сменяющееся постепенным возрастанием в ходе фазы $\mathrm{S}$ и достижением максимума к следующему митозу [32]. Для многих злокачественных опухолей высокий индекс пролиферативной активности Кi-67 связан с агрессивностью опухоли и, как правило, неблагоприятным прогнозом для выживаемости пациентов [33]. В международных базах данных PubMed, Medline и eLIBRARY нами найдены единичные сообщения, посвященные изучению зависимости между значениями ИКД и индексом Кі-67 различных экстра- и интракраниальных опухолей. Так, в работе G. Zheng с соавт. [34] отмечено, что у пациентов с диагнозом рака желудка имеет место достоверная отрицательная корреляционная зависимость между значениями ИКД и индексом Кi-67. Z. Huang с соавт. [35] подтверждают указанные данные в отношении пациентов с гепатоцеллюлярной карциномой. Для опухолей головного мозга зависимость между значениями ИКД и индексом Кi-67 является неоднозначной. К примеру, некоторые авторы $[9,10]$ в своих исследованиях отмечают статистически значимую корреляцию между значениями ИКД и индексом Кi-67 как в группе менингиом $\mathrm{G}=\mathrm{I}$, так и в группах менингиом $\mathrm{G}=\mathrm{II}$ и $\mathrm{G}=\mathrm{III}$. С другой стороны, в сообщении D. Ginat c соавт. [11] достоверной корреляции между ИКД и индексом Ki67 для менингиом $\mathrm{G}=$ III не отмечено. Z. Fatima с соавт. [12] в своем наблюдении также не отметили достоверной корреляции между значением ИКД и индексом Кi-67. Подобных исследований в отношении метастатических опухолей головного мозга нами не найдено. Согласно собственным данным, для метастазов в головной мозг отмечена достоверная обратная корреляционная зависимость между значениями ИКД и индексом пролиферативной активности Ki-67 ( $r=-0,774 ; p=0,014)$.

На сегодняшний день известно несколько работ, посвященных изучению зависимости между значениями ИКД злокачественных опухолей и общей выживаемостью пациентов. Так, в клинической серии L. Curvo-Semedo с соавт. [22] показано, что низкие значения ИКД при колоректальном раке ассоциированы с высокой степенью агрессивности опухоли, плохим ответом пациентов на проводимую терапию и низкой общей выживаемостью. Аналогичные результаты получены при исследовании данных параметров у пациентов с диагнозом рака легкого 
и молочной железы [20, 21]. В случае ряда опухолей головного мозга (лимфомы и макроаденомы гипофиза) полученные высокие значения ИКД являются неблагоприятными прогностическими факторами для выживаемости данной группы пациентов [13, 14]. С другой стороны, в исследовании R. Zakaria с соавт. [15] наглядно продемонстрировано, что при значении ИКД метастатических опухолей головного мозга более 919,4 мм² $^{2}$ сек отмечается достоверное увеличение общей выживаемости пациентов - 9,7 мес (95\% ДИ 8,5-11,0) против 6,2 мес (95\% ДИ $3,7-8,8 ; p=0,049)$. C.-C. Lee с соавт. [16] в своей клинической серии из 42 пациентов с метастазами в головной мозг не получили достоверных различий в общей выживаемости больных с различными значениями ИКД после проведенных курсов радиохирургии. В нашем исследовании выявлена статистически значимая корреляционная зависимость между значениями ИКД и общей выживаемостью пациентов с метастазами в головной мозг, что согласуется с некоторыми литературными данными. При этом общая выживаемость пациентов со значением ИКД опухоли более 947,2 мм²/сек составила 9,8 мес (95\% ДИ $8,6-11,3)$, а при ИКД метастазов в головной мозг менее $947,2 \mathrm{мм}^{2} /$ сек - 6,4 мес (95\% ДИ 3,7-9,1).

\section{Ограничения исследования}

Настоящее исследование имеет ряд ограничений, которые необходимо обозначить. Исследование имеет ретроспективный характер и основано на данных, полученных в одном центре с малым количеством исследуемых пациентов, что не могло не отразиться на мощности статистического анализа. Кроме того, значения ИКД, полученные в нашей клинической серии, сложно сравнивать с результатами других исследований, что связано с использованием различных аппаратов МРТ и программ для подсчета ИКД.

\section{Заключение}

Проведенное исследование продемонстрировало достоверную корреляционную зависимость между индексом пролиферативной активности Кі-67 и ИКД метастатических опухолей головного мозга. Кроме того, ИКД является статистически значимым прогностическим фактором для общей выживаемости пациентов с метастазами в головной мозг. Безусловно, требуется проведение дальнейших крупных мультицентровых исследований на большем количестве пациентов с исследованием различных гистологических типов метастазов в головной мозг, а также применение унифицированных протоколов подсчета ИКД для данной группы опухолей головного мозга.

\section{Источник финансирования}

Исследование выполнено при поддержке гранта Российского научного фонда (проект № 14-32-00006).

\section{Конфликт интересов}

Авторы данной статьи подтвердили отсутствие конфликта интересов, о котором необходимо сообщить.

\section{ЛИТЕРАТУРА}

1. Caffo M, Barresi V, Caruso G, et al. Innovative therapeutic strategies in the treatment of brain metastases. Int $J$ Mol Sci. 2013;14(1):2135-2174. doi: 10.3390/ijms14012135.

2. Бывальцев В.А., Сороковиков В.А., Панасенков С.Ю., и др. Редкий случай интравентрикулярного рецидива метастаза меланомы, удаленного с использованием эндоскопической ассистенции // Вопросы нейрохирургии им. Н.Н. Бурденко. 2010. - №2 - C. 29-33. [Byvaltsev VA, Sorokovikov VA, Panasenkov $\mathrm{SYu}$, et al. A rare case of intraventricular recurrence of melanoma metastasis treated by endoscope-assisted surgery. Zh Vopr Neirokhir Im NN Burdenko. 2010;(2):29-32. (In Russ).].

3. Kyritsis AP, Markoula S, Levin VA. A systematic approach to the management of patients with brain metastases of known or unknown primary site. Cancer Chemother Pharmacol. 2012;69(1):1-13. doi: 10.1007/s00280-011-1775-9.

4. El-Habashy SE, Nazief AM, Adkins CE, et al. Novel treatment strategies for brain tumors and metastases. Pharm Pat Anal. 2014;3(3):279-296. doi: 10.4155/ppa.14.19.

5. Egelhoff JC, Ross JS, Modic MT, et al. MR imaging of metastatic GI adenocarcinoma in brain. AJNR Am J Neuroradiol. 1992;13(4):1221-1224.

6. Carrier DA, Mawad ME, Kirkpatrick JB, Schmid MF. Metastatic adenocarcinoma to the brain: MR with pathologic correlation. AJNR Am J Neuroradiol. 1994;15(1):155-159.

7. Duygulu G, Ovali GY, Calli C, et al. Intracerebral metastasis showing restricted diffusion: correlation with histopathologic findings. Eur J Radiol. 2010;74(1):117-120. doi: 10.1016/j.ejrad.2009.03.004.

8. Hayashida Y, Hirai T, Morishita S, et al. Diffusion-weighted imaging of metastatic brain tumors: comparison with histologic type and tumor cellularity. AJNR Am J Neuroradiol. 2006;27(7):1419-1425.

9. Tang Y, Dundamadappa SK, Thangasamy S, et al. Correlation of apparent diffusion coefficient with $\mathrm{Ki}-67$ proliferation index in grading meningioma. AJR Am J Roentgenol. 2014;202(6):1303-1308. doi: 10.2214/AJR.13.11637.

10. Бывальцев В.А., Степанов И.А., Кичигин А.И., Антипина С.Л. Возможности диффузионно-взвешенной МРТ в дифференциальной диагностике степени злокачественности менингиом головного мозга // Сибирский онкологический журнал. - 2017. T.16. - №3 - C. 19-26. [Byvaltsev VA, Stepanov IA, Kichigin AI, Antipina SL. Diffusion-weighted MRI in the differential diagnosis of brain meningiomas. Siberian journal of oncology. 2017;16(3):19-26. (In Russ).] doi: 10.21294/1814-4861-2017-16-3-19-26.

11. Ginat DT, Mangla R, Yeaney G, Wang HZ. Correlation of diffusion and perfusion MRI with Ki-67 in high-grade meningiomas. AJR Am J Roentgenol. 2010;195(6):1391-1395. doi: 10.2214/AJR.10.4531.

12. Fatima $\mathrm{Z}$, Motosugi $\mathrm{U}$, Waqar $\mathrm{AB}$, et al. Associations among q-space MRI, diffusion-weighted MRI and histopathological parameters in meningiomas. Eur Radiol. 2013;23(8):2258-2263. doi: 10.1007/s00330-013-2823-0.

13. Boxerman JL, Rogg JM, Donahue JE, et al. Preoperative MRI evaluation of pituitary macroadenoma: imaging features predictive of successful transsphenoidal surgery. AJR Am J Roentgenol. 2010;195(3):720-728. doi: 10.2214/Ajr.09.4128.

14. Barajas RF Jr, Rubenstein JL, Chang JS, et al. Diffusion-weighted MR imaging derived apparent diffusion coefficient is predictive of clinical outcome in primary central nervous system lymphoma. AJNR Am J Neuroradiol. 2010;31(1):60-66. doi: 10.3174/ajnr.A1750.

15. Zakaria R, Das K, Radon M, et al. Diffusion-weighted MRI characteristics of the cerebral metastasis to brain boundary predicts patient outcomes. BMC Med Imaging. 2014;14:26. doi: 10.1186/1471-2342-14-26.

16. Lee CC, Wintermark M, Xu ZY, et al. Application of diffusionweighted magnetic resonance imaging to predict the intracranial metastatic tumor response to gamma knife radiosurgery. J Neurooncol. 2014;118(2):351-361. doi: 10.1007/s11060-014-1439-9. 
17. Тоноян А.С., Пронин И.Н., Пицхелаури Д.И., и др. Диффузионно-куртозисная МРТ в диагностике злокачественности глиом головного мозга // Медицинская визуализация. - 2015. №1 - C. 7-18. [Tonoyan AS, Pronin IN, Pitskhelauri DI, et al. Diffusion kurtosis imaging in diagnostics of brain glioma malignancy. Meditsinskaya vizualizatsiya. 2015;(1):7-18. (In Russ).]

18. Byvaltsev VA, Stepanov IA, Kalinin AA, Shashkov KV. Diffusionweighted magnetic resonance tomography in the diagnosis of intervertebral disk degeneration. Biomed Eng (NY). 2016;50(4):253-256. doi: 10.1007/s10527-016-9632-0.

19. Пронин И.Н., Тоноян А.С., Шульц Е.И., и др. Диффузионнокуртозисная МРТ в оценке Ki-67/MIB-1 LI глиальных опухолей // Медицинская визуализация. - 2016. - №5 - С. 6-17. [Pronin IN, Tonoyan AS, Shults EI, et al. Diffusion kurtosis MRI in assesment of Ki-67/MIB-1 LI in gliomas. Meditsinskaya vizualizatsiya. 2016;(5):6-17. (In Russ).]

20. Nakajo M, Kajiya Y, Kaneko T, et al. FDG PET/CT and diffusion-weighted imaging for breast cancer: prognostic value of maximum standardized uptake values and apparent diffusion coefficient values of the primary lesion. Eur J Nucl Med Mol Imaging. 2010;37(11):2011-2020. doi: 10.1007/s00259-010-1529-7.

21. Ohno Y, Koyama H, Yoshikawa T, et al. Diffusion-weighted MRI versus $18 \mathrm{~F}$-FDG PET/CT: performance as predictors of tumor treatment response and patient survival in patients with non-small cell lung cancer receiving chemoradiotherapy. AJR Am J Roentgenol. 2012;198(1):75-82. doi: 10.2214/AJR.11.6525.

22. Curvo-Semedo L, Lambregts DMJ, Maas M, et al. Diffusionweighted MRI in rectal cancer: apparent diffusion coefficient as a potential noninvasive marker of tumor aggressiveness. J Magn Reson Imaging. 2012;35(6):1365-1371. doi: 10.1002/jmri.23589.

23. Teixidor P, Arráez MÁ, Villalba G, et al. Safety and efficacy of 5 -aminolevulinic acid for high grade glioma in usual clinical practice: a prospective cohort study. PLoS One. 2016;11(2):e0149244. doi: 10.1371/journal.pone.0149244.

24. Abercrombie M. Estimation of nuclear population from microtome sections. Anat Rec. 1946;94:239-247. doi: 10.1002/ar.1090940210.

25. Cox DR, Snell EJ. Analysis of binary data. 2nd ed. London, UK: Chapman \& Hall; 1989.
26. Kaplan EL, Meier P. Nonparametric estimation from incomplete observations. J Am Stat Assoc. 1958;53(282):457-481. doi: $10.2307 / 2281868$

27. Ramli N, Khairy AM, Seow P, et al. Novel application of chemical shift gradient echo in- and opposed-phase sequences in $3 \mathrm{~T}$ MRI for the detection of H-MRS visible lipids and grading of glioma. European Radiology. 2016;26:2019-2029. doi: 10.1007/s00330-015-4045-0.

28. Sugahara T, Korogi Y, Kochi M, et al. Usefulness of diffusionweighted MRI with echo-planar technique in the evaluation of cellularity in gliomas. J Magn Reson Imaging. 1999;9(1):5360. doi: 10.1002/(SICI)1522-2586(199901)9:1<53::AIDJMRI7>3.0.CO;2-2.

29. Fan GG, Deng QL, Wu ZH, Guo QY. Usefulness of diffusion/ perfusion-weighted MRI in patients with non-enhancing supratentorial brain gliomas: a valuable tool to predict tumour grading? $\mathrm{Br} J$ Radiol. 2006;79(944):652-658. doi: 10.1259/bjr/25349497.

30. Langley RR, Fidler IJ. The seed and soil hypothesis revisited — the role of tumor-stroma interactions in metastasis to different organs. Int J Cancer. 2011;128(11):2527-2535. doi: 10.1002/ijc.26031.

31. Moorman AM, Vink R, Heijmans HJ, et al. The prognostic value of tumour-stroma ratio in triple-negative breast cancer. Eur J Surg Oncol. 2012;38(4):307-313. doi: 10.1016/j.ejso.2012.01.002.

32. Inwald EC, Klinkhammer-Schalke M, Hofstadter F, et al. Ki-67 is a prognostic parameter in breast cancer patients: results of a large population-based cohort of a cancer registry. Breast Cancer Res Treat. 2013;139(2):539-552. doi: 10.1007/s10549-013-2560-8.

33. Rosell R, Tian Y, Ma Z, et al. Clinicopathological and prognostic value of Ki-67 expression in bladder cancer: a systematic review and meta-analysis. PLoS One. 2016;11(7):e0158891. doi: 10.1371/ journal.pone.0158891.

34. Zheng G, Cheng X, Wang L, et al. [Correlation of MRI apparent diffusion coefficient with molecular marker Ki-67 in gastric cancer. (In Chinese).] Zhonghua Wei Chang Wai Ke Za Zhi. 2017;20(7):803-808.

35. Huang ZQ, Xu XQ, Meng XJ, et al. Correlations between ADC values and molecular markers of Ki-67 and HIF-1 alpha in hepatocellular carcinoma. Eur J Radiol. 2015;84(12):2464-2469. doi: 10.1016/j.ejrad.2015.09.013.

\section{КОНТАКТНАЯ ИНФОРМАЦИЯ}

Бывальцев Вадим Анатольевич, доктор медицинских наук, главный нейрохирург Дирекции здравоохранения ОАО «РЖД»; руководитель Центра нейрохирургии Дорожной клинической больницы на ст. Иркутск-Пассажирский ОАО «РЖД-Медицина»; заведующий курсом нейрохирургии Иркутского государственного медицинского университета; заведующий научно-клиническим отделом нейрохирургии и ортопедии Иркутского научного центра хирургии и травматологии; профессор кафедры травматологии, ортопедии и нейрохирургии Иркутской государственной медицинской академии последипломного образования; ведущий научный сотрудник Института ядерной физики им. Г.И. Будкера

Адрес: 664082, Иркутск, ул. Боткина, д. 10, тел.: +7 (3952) 63-85-28, e-mail: byval75vadim@yandex.ru, SPIN-код: 5996-6477, ORCID: http://orcid.org/0000-0003-4349-7101

Степанов Иван Андреевич, аспирант курса нейрохирургии Иркутского государственного медицинского университета Адрес: 664003, Иркутск, ул. Красного Восстания, д. 14, тел.: +7 (3952) 63-88-30, e-mail: edmoilers@mail.ru, SPIN-код: 5485-5316, ORCID: http://orcid.org/0000-0001-9039-9147

Кичигин Александр Иванович, аспирант курса нейрохирургии Иркутского медицинского университета, стажерисследователь Института ядерной физики им. Г.И. Будкера СО РАН Адрес: 664003, Иркутск, ул. Боткина, д. 10, тел.: +7 (3952) 63-85-78, e-mail: sam@211.ru, SPIN-код: 4321-2422, ORCID: https://orcid.org/0000-0001-8763-2905

Каныгин Владимир Владимирович, врач-нейрохирург, кандидат медицинских наук, доцент, заведующий лабораторией медико-биологических проблем бор-нейтронзахватной терапии Новосибирского государственного университета

Адрес: 630090, Новосибирск, ул. Пирогова, д. 2, тел.: +7 (3833) 63-43-33, e-mail: kanigin@mail.ru, SPIN-код: 4211-2417, ORCID: https://orcid.org/0000-0003-3533-6076

Ступак Вячеслав Владимирович, доктор медицинских наук, профессор, руководитель клиники нейрохирургии Новосибирского научно-исследовательского института травматологии и ортопедии им. Я.Л. Цивьяна Адрес: 630091, Новосибирск, ул. Фрунзе, д. 17, тел.: +7 (3833) 63-31-31, e-mail: vstupak@niito.ru, SPIN-код: 4111-2527, ORCID: https://orcid.org/0000-0002-6074-6248 